\title{
Factors Associated With Delays in Chemotherapy Initiation Among Patients With Breast Cancer at a Comprehensive Cancer Center
}

\author{
Katya Losk, $\mathrm{MPH}^{\mathrm{a}}$; Ines Vaz-Luis, MD, MSc ${ }^{\mathrm{a}}$; Kristen Camuso, MPH ${ }^{\mathrm{b}}$; Rafael Batista, MDc; Max Lloyd ${ }^{\mathrm{a}}$; \\ Mustafa Tukenmez, MD ; Mehra Golshan, MD; Nancy U. Lin, MD; and Craig A. Bunnell, MD, MPH, MBA ${ }^{a}$
}

\begin{abstract}
Background: National guidelines endorse time-dependent quality metrics for breast cancer care. We examined factors associated with delays in chemotherapy initiation at an NCl-Designated Comprehensive Cancer Center. Patients and Methods: We identified 523 patients who received postoperative adjuvant chemotherapy between January 2011 and December 2013 at our center. We defined 28 days from last definitive surgery (LDS) to chemotherapy as the target time frame, and an unacceptable delay in chemotherapy initiation (UCD) as greater than 42 days from LDS. Multivariate regression models were used to identify factors associated with UCD and the impact of Oncotype DX testing in patients with hormone receptor (HR)-positive breast cancer. Results: Median days between LDS and chemotherapy initiation was 34 (interquartile range, 15), with $30 \%$ of patients starting within 28 days of LDS and $26.9 \%$ having UCD. Tumor characteristics such as subtype and stage affected UCD; patients with HR-positive or HER2-positive tumors were more likely to be delayed compared with those with triple-negative breast cancer. Patients with stage I disease, those undergoing mastectomy with or without immediate reconstruction, and those whose pathology sign-out was greater than 10 days postoperatively were more likely to be delayed. A higher proportion of UCD was found in HR-positive patients (31\%) for whom Oncotype DX testing was ordered compared with those in whom it was not ordered (20\%). Conclusions: This study provides insight into subpopulations that may be at risk to experience delays in chemotherapy initiation, directing interventions to improve the timeliness of care.
\end{abstract}

J Natl Compr Canc Netw 2016;14(12):1519-1526

\section{Background}

Over the past 50 years, substantial reductions in early breast cancer mortality have been documented due to a combination of earlier detection and to advances in adjuvant treatment. ${ }^{1-4}$ In particular, adjuvant chemotherapy has been shown to decrease the risk of breast cancer recurrence, with an estimated relative risk reduction of up to $30 \%$ to $50 \%$. 5,6

The benefit of adjuvant chemotherapy may partly depend on the timing of therapy initiation. Recent

From a Department of Medical Oncology and ' $\mathrm{Q}$ Quality and Patient Safety, Dana-Farber Cancer Institute, Boston, Massachusetts; 'Oncoclinicas, Sao Paulo, Brazil; 'Department of General Surgery, Istanbul Medical School, Istanbul, Turkey; and 'Department of Surgery, Dana-Farber Cancer Institute/Brigham and Women's Hospital, Boston, Massachusetts. Submitted June 1, 2016; accepted for publication August 8, 2016. studies suggest that delays in adjuvant chemotherapy initiation greater than 49 to 90 days after surgery may be associated with inferior outcomes, particularly among patients with triple-negative breast cancer (TNBC), HER2-positive subtypes, and higher-stage tumors..$^{7-12}$ A meta-analysis conducted by $\mathrm{Yu}$ et $\mathrm{al}^{13}$ suggested that overall survival decreases by $15 \%$ for every 4 -week delay in chemotherapy initiation. Although these retrospective data must be interpreted carefully given potential confounders and selection bias, from a patient-centered perspective, delays to chemotherapy initiation also can
The authors have disclosed that they have no financial interests, arrangements, affiliations, or commercial interests with the manufacturers of any products discussed in this article or their competitors. This initiative was supported in part by the NCCN Opportunities for Improvement grant. Correspondence: Craig A. Bunnell, MD, MPH, MBA, Dana-Farber Cancer Institute, 450 Brookline Avenue, Boston, MA 02215.

E-mail: craig_bunnell@dfci.harvard.edu 
adversely impact patient satisfaction and increase stress and anxiety.

Limited knowledge exists on processes of care within institutional and physician control, which influence the timing of chemotherapy initiation. These processes include referral from surgery to medical oncology, postoperative appointment scheduling, pathology turnaround time, and the role of additional testing, such as Oncotype DX (Genomic Health, Redwood City, CA). In this study, we sought to elucidate factors associated with delays in chemotherapy initiation after last definitive surgery (LDS), and to characterize the role of these factors on the treatment timeline for patients treated at our comprehensive cancer center.

\section{Patients and Methods}

\section{Setting}

We examined the care of women treated for breast cancer at the Dana-Farber/Brigham and Women's Cancer Center (DF/BWCC), a Harvard-affiliated NCI-Designated Comprehensive Cancer Center. The breast cancer program is organized as a multidisciplinary disease center. With more than 3,000 new patients annually, the DF/BWCC breast cancer program involves 28 medical, 12 surgical, and 4 radiation oncologists.

\section{Data Source}

We developed an internal dataset of patients treated for breast cancer at our institution through the integration of administrative and billing databases from the Brigham and Women's Hospital and the Dana-Farber Cancer Institute. ${ }^{14}$ Using ICD-9 and CPT codes from surgical billing data, women with breast cancer who underwent lumpectomy, mastectomy, or mastectomy with immediate reconstruction (MIR) at our 2 primary surgical sites between January 2011 and December 2013 were identified. Using unique patient identifiers, we extracted all adjuvant chemotherapy administered to this surgical cohort through our chemotherapy order entry system.

This initiative was undertaken as a quality improvement project and was determined to be exempt from review by the Dana-Farber/Harvard Cancer Center Institutional Review Board.

\section{Study Cohort}

We identified a cohort of 523 women with invasive stage I-III breast cancer who underwent surgery at 1 of 2 surgical sites and initiated adjuvant chemotherapy between January 2011 and December 2013. Patients who received neoadjuvant or investigational therapy or preoperative radiation therapy, patients with stage IV disease, and male patients were excluded.

\section{Outcome Measures}

Our primary outcome of interest was the interval between LDS to chemotherapy initiation. LDS was defined as the date of the breast surgical procedure immediately before chemotherapy initiation. Through a multidisciplinary consensus process, a team of medical, surgical, and radiation oncologists determined that the target for initiation of chemotherapy should be 28 or fewer days from LDS, and the unacceptable delay to chemotherapy initiation (UCD) should be 42 or more days from LDS, to ensure the optimal patient care experience. The target of 28 or fewer days was used to distinguish timely from delayed care in our subsequent analyses. Wait times to chemotherapy initiation were grouped as follows: 28 or fewer days, 29 to 41 days, and 42 or more days.

\section{Covariates}

Covariates of interest were: race (white vs nonwhite), age at LDS (<40, 40-49, 50-59, 60-69, $\geq 70$ years), insurance (private vs public), year of surgery (2011, 2012, 2013), stage (I, II, III), grade (1, 2, 3 ), breast cancer subtypes (estrogen receptor [ER]positive and/or progesterone receptor $[\mathrm{PR}]$-positive/ HER2-negative; HER2-positive; TNBC), surgery type (lumpectomy, mastectomy alone, MIR), Oncotype DX testing (yes vs no), pathology turnaround time ( $\leq 10$ days from LDS vs $>10$ days after LDS). Pathology sign-out more than 10 days from LDS was considered "delayed."

The aggregated data set included the following variables: LDS date, LDS procedure type, date of postoperative medical oncology appointment, adjuvant chemotherapy start date, and patient sociodemographic characteristics. Medical chart reviews were conducted to extract information on pathology sign-out date, pathologic stage, tumor grade, tumor subtype, and use of Oncotype DX testing. 
Delays to Chemotherapy in Breast Cancer

\section{Statistical Analysis}

Descriptive statistics were generated on the time from LDS to chemotherapy initiation. A series of multivariate multinomial logistic regressions were performed to evaluate predictors of timely chemotherapy initiation. In these models, we compared the likelihood of waiting 29 to 41 days to initiate chemotherapy versus starting 28 or fewer days after LDS, and the probability of waiting 42 or more days versus initiating chemotherapy at 28 or fewer days after LDS. In the first model we examined factors associated with delay, including patient age, race, insurance, year of surgery, stage, tumor subtype, LDS procedure, and pathology turnaround time. In the second model we examined predictors of timely chemotherapy initiation for patients with hormone receptor (HR)-positive/HER2negative disease. In this model, we assessed for all factors included in the first model with the addition of whether Oncotype DX testing was performed. Models focused on TNBC and HER2-positive disease were not performed due to smaller patient populations. In our third model, we assessed factors associated with timely chemotherapy in patients with stage II and III disease. Factors included in this model were consistent with the first model. Patients who initiated chemotherapy 28 or fewer days after LDS served as the reference cohort for all 3 models. Adjusted odds ratios (ORs), 95\% confidence intervals (CIs), and $P$ values were calculated to ascertain the strength of the association between each variable and delayed care. For patients who initiated chemotherapy 42 or more days from LDS, chart reviews were conducted to determine the presence of additional factors, including postoperative surgical complications, consideration of clinical trial enrollment, and patient-driven decision-making.

\section{Results}

\section{Cohort Characteristics}

We identified 523 women with stage I-III breast cancer who initiated chemotherapy between 2011 and 2013 after breast surgery at DF/BWCC. The mean age was 51.1 years; $81.1 \%$ were white; $95.2 \%$ were English-speaking; and $80.1 \%$ had private insurance. Approximately half of the patients underwent lumpectomy (50.7\%), 17.0\% had mastectomy alone, and $32.3 \%$ underwent MIR. Patient and tumor characteristics are listed in Table 1.

\section{Time From LDS to Chemotherapy}

Overall, the median time to initiate chemotherapy was 34.0 days (interquartile range [IQR], 15.0), ranging from 6 to 132 days (Figure 1). Among these patients, $30.5 \%$ initiated chemotherapy within 28 days of LDS, $42.4 \%$ initiated chemotherapy between 29 and 41 days, and $26.9 \%$ experienced UCD.

Multivariate multinomial analysis in Table 2 highlights factors associated with the timeliness of receiving chemotherapy. When comparing patients with UCD versus those who started chemotherapy 28 or fewer days after LDS, older patients (60-69 vs 40-49 years: OR 2.92; 95 CI, 1.34-6.36) were significantly more likely to experience UCD compared with younger patients. Patients with HR-positive/ HER2-negative disease (HR-positive/HER2-negative vs HR-negative/HER2-negative: OR, 2.71; 95 CI, 1.28-5.73) were significantly more likely to experience UCD compared with those with TNBC. Both mastectomy alone and MIR were found to be significantly associated with delayed receipt of chemotherapy (mastectomy vs lumpectomy: OR, 3.32; 95 CI, 1.68-6.56; MIR vs lumpectomy: OR, 5.09; 95 CI, 2.77-9.34). Patients whose pathology sign-out was 10 or more days from LDS (pathology sign-out $>10$ days vs within 10 days: OR, 2.04; 95 CI, 1.193.50 ) were more likely to experience UCD compared with patients whose pathology sign-out was 10 or fewer days from LDS.

Similar trends were observed among patients with HR-positive/HER2-negative disease (Table 3). In addition, patients for whom Oncotype DX testing was ordered were more likely to wait 29 to 41 days (yes vs no: OR, 3.41; 95 CI, 1.32-8.85) and experience UCD (yes vs no: OR, 7.48; 95 CI, 2.67-20.94) compared with those who did not receive this test.

Finally, when we restricted the analyses for patients with more advanced disease (stage II or III), the only factor that remained significant was surgical procedure (Table 4). Patients who underwent MIR (MIR vs lumpectomy: OR, 3.87; 95 CI, 1.76-8.49) were significantly more likely to experience UCD compared with those undergoing lumpectomy.

\section{Reasons for UCD}

Among the 141 patients who experienced UCD, 23 had postoperative wound complications and 12 were considering a clinical trial. Other reasons for UCD included family events, comorbidities, and patient deci- 
Losk et al

\begin{tabular}{|c|c|c|c|c|c|}
\hline & $\begin{array}{c}\text { All Patients, } n \\
(\%) \\
(\mathrm{N}=523) \\
\end{array}$ & $\begin{array}{c}\leq 28 \text { Days, } n \\
(\%) \\
(\mathrm{N}=160) \\
\end{array}$ & $\begin{array}{c}29-41 \text { Days, } n \\
(\%) \\
(\mathrm{N}=22 \\
\end{array}$ & $\begin{array}{c}\geq 42 \text { Days, } \mathrm{n} \\
(\%) \\
(\mathrm{N}=141)\end{array}$ & $P$ Value \\
\hline \multicolumn{6}{|l|}{ Age, y } \\
\hline$<40$ & $68(13.0 \%)$ & $29(18.1 \%)$ & $24(10.8 \%)$ & $15(10.6 \%)$ & \multirow[t]{5}{*}{.04} \\
\hline $40-49$ & $165(31.5 \%)$ & $51(31.9 \%)$ & $79(35.6 \%)$ & $35(24.8 \%)$ & \\
\hline $50-59$ & $150(28.7 \%)$ & $48(30.0 \%)$ & $58(26.1 \%)$ & $44(31.2 \%)$ & \\
\hline $60-69$ & $113(21.6 \%)$ & $24(15.0 \%)$ & $53(23.9 \%)$ & $36(25.6 \%)$ & \\
\hline$\geq 70$ & $27(5.2 \%)$ & $8(5.0 \%)$ & $8(3.6 \%)$ & $11(7.8 \%)$ & \\
\hline \multicolumn{5}{|l|}{ Race } & \multirow[t]{4}{*}{.17} \\
\hline White & $424(81.1 \%)$ & $123(76.9 \%)$ & $191(86.0 \%)$ & $110(78.0 \%)$ & \\
\hline Nonwhite & $79(15.1 \%)$ & $28(17.5 \%)$ & $26(11.7 \%)$ & $25(17.7 \%)$ & \\
\hline Missing & $20(3.8 \%)$ & $9(5.6 \%)$ & $5(2.3 \%)$ & $6(4.3 \%)$ & \\
\hline \multicolumn{5}{|l|}{ Primary insurance } & \multirow[t]{5}{*}{11} \\
\hline Private & $413(79.0 \%)$ & $132(82.5 \%)$ & $180(81.1 \%)$ & $101(71.6 \%)$ & \\
\hline Medicare & $81(15.5 \%)$ & $21(13.1 \%)$ & $28(12.6 \%)$ & $32(22.7 \%)$ & \\
\hline Medicaid & $23(4.4 \%)$ & $6(3.8 \%)$ & $12(5.4 \%)$ & $5(3.6 \%)$ & \\
\hline Other & $6(1.1 \%)$ & $1(0.6 \%)$ & $2(0.9 \%)$ & $3(2.1 \%)$ & \\
\hline \multicolumn{5}{|l|}{ Primary language } & \multirow[t]{3}{*}{.94} \\
\hline English & $498(95.2 \%)$ & $152(95.0 \%)$ & $211(95.1 \%)$ & $135(95.7 \%)$ & \\
\hline Non-English & $25(4.8 \%)$ & $8(5.0 \%)$ & $11(4.9 \%)$ & $6(4.3 \%)$ & \\
\hline \multicolumn{5}{|l|}{ Year of surgery } & \multirow[t]{4}{*}{.45} \\
\hline 2011 & $111(21.2 \%)$ & $28(17.4 \%)$ & $50(22.5 \%)$ & $33(23.4 \%)$ & \\
\hline 2012 & $207(39.6 \%)$ & $66(41.3 \%)$ & $92(41.5 \%)$ & $49(34.8 \%)$ & \\
\hline 2013 & $205(39.2 \%)$ & $66(41.3 \%)$ & $80(36.0 \%)$ & $59(41.8 \%)$ & \\
\hline \multicolumn{5}{|l|}{ Stage } & \multirow[t]{4}{*}{.60} \\
\hline 1 & $215(41.1 \%)$ & $71(44.4 \%)$ & $87(39.2 \%)$ & $57(40.4 \%)$ & \\
\hline II & $238(45.5 \%)$ & $68(42.5 \%)$ & $101(45.5 \%)$ & $69(48.9 \%)$ & \\
\hline III & $70(13.4 \%)$ & $21(13.1 \%)$ & $34(15.3 \%)$ & $15(10.7 \%)$ & \\
\hline \multicolumn{5}{|l|}{ Grade } & \multirow[t]{4}{*}{.15} \\
\hline 1 & $19(3.6 \%)$ & $5(3.1 \%)$ & $8(3.6 \%)$ & $6(4.3 \%)$ & \\
\hline 2 & $181(34.6 \%)$ & $43(26.9 \%)$ & $84(37.8 \%)$ & $54(38.3 \%)$ & \\
\hline 3 & $323(61.8 \%)$ & $112(70.0 \%)$ & $130(58.6 \%)$ & $81(57.4 \%)$ & \\
\hline \multicolumn{5}{|l|}{ Tumor subtype } & \multirow[t]{4}{*}{.05} \\
\hline HR+/HER2- & $263(50.3 \%)$ & $67(41.9 \%)$ & $122(55.0 \%)$ & $74(52.5 \%)$ & \\
\hline HER2+ & $156(29.8 \%)$ & $52(32.5 \%)$ & $58(26.1 \%)$ & $46(32.6 \%)$ & \\
\hline HR-/HER2- & $104(19.9 \%)$ & $41(25.6 \%)$ & $42(18.9 \%)$ & $21(14.9 \%)$ & \\
\hline \multicolumn{5}{|l|}{ Type of surgery } & \multirow[t]{4}{*}{$<.0001$} \\
\hline Lumpectomy & $265(50.7 \%)$ & $101(63.1 \%)$ & $116(52.3 \%)$ & $48(34.0 \%)$ & \\
\hline Mastectomy & $89(17.0 \%)$ & $23(14.4 \%)$ & $32(14.4 \%)$ & $34(24.1 \%)$ & \\
\hline $\begin{array}{l}\text { Mastectomy with } \\
\text { reconstruction }\end{array}$ & $169(32.3 \%)$ & $36(22.5 \%)$ & $74(33.3 \%)$ & $59(41.8 \%)$ & \\
\hline \multicolumn{5}{|l|}{ Pathology sign-out } & \multirow[t]{3}{*}{.007} \\
\hline Within 10 days & $366(70.0 \%)$ & $123(76.9 \%)$ & $158(71.2 \%)$ & $85(60.3 \%)$ & \\
\hline$>10$ days & $157(30.0 \%)$ & $37(23.1 \%)$ & $64(28.8 \%)$ & $56(39.7 \%)$ & \\
\hline
\end{tabular}

sion-making. In half of the patients $(n=71)$, we were not able to ascertain any additional factors that may have played a role in delaying chemotherapy initiation.

\section{Discussion}

Within a cohort of more than 500 consecutive women with breast cancer treated with adjuvant chemotherapy, the median number of days between LDS and chemotherapy initiation was 34 , with most patients initiating treatment within 6 weeks of LDS. However, $26.9 \%$ of patients experienced an unacceptable delay, as defined prospectively by our breast cancer clinical leadership based on internal time-totreatment targets established in our clinical practice. We found several factors associated with delays to chemotherapy, including age, tumor subtype, surgical procedure, and pathology turnaround time. 
Delays to Chemotherapy in Breast Cancer

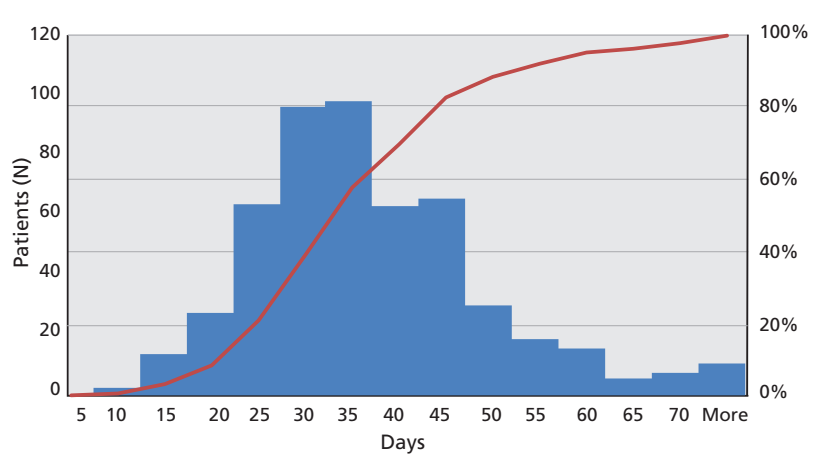

Figure 1. Days between last definitive surgery and chemotherapy initiation.

In patients with HR-positive tumors, Oncotype DX testing significantly increased the likelihood of UCD. When focusing on patients with higher-stage disease, only surgical procedure remained associated with timing of chemotherapy.

Although the optimal timing of chemotherapy initiation is not clearly defined, ESMO clinical practice guidelines recommend commencing treatment within 2 to 6 weeks of surgery, ${ }^{15}$ and ASCO/NCCN recommend adjuvant chemotherapy initiation within 12 weeks of initial diagnosis for women younger than 70 years with stage II or III HR-negative breast cancer as a quality measure. ${ }^{16}$ These recommendations are based on a combination of expert opinion and evidence from retrospective studies, because ethical imperatives prevent conducting prospective randomized trials testing the effect of purposeful delays on clinical outcomes.

Retrospective studies examining the impact of adjuvant chemotherapy wait times on survival and patient outcomes have had mixed results, which may be attributed to differences in patient populations, disease-specific factors, variability in the adjuvant treatment administered, and overall patient health status. $^{7-12,17,18}$ However, emerging data suggest that, particularly for patients at higher risk of recurrence, including those with higher-stage disease, HER2positive or TNBC subtypes, or a high proliferative rate, delayed time from surgery to chemotherapy can adversely impact outcomes. ${ }^{6-11}$ Gagliato et $\mathrm{al}^{10}$ found that patients with stage II disease experienced an $18 \%$ increase in the risk of distant relapse-free survival when treatment was initiated 31 to 60 days after surgery (hazard ratio, 1.18; 95\% CI, 1.03-1.34) compared with when therapy was initiated within 30 days of surgery. Among patients with stage III breast cancer, waiting more than 60 days to initiate che- motherapy led to a $36 \%$ increase in the risk of distant relapse (hazard ratio, 1.34; 95\% CI, 1.02-1.80) compared with when therapy was initiated within 30 days of surgery. Farolfi et $\mathrm{al}^{9}$ identified that wait times greater than 49 days led to inferior overall survival among patients with rapidly proliferating tumors $(P=.043)$. A third study, conducted by Chavez-MacGregor et $\mathrm{al},{ }^{7}$ found that patients treated 91 or more days from surgery experienced worse overall survival (hazard ratio, 1.34; 95\% CI, 1.17-2.00) and worse breast cancer-specific survival (hazard ratio, 1.27; 95\% CI, 1.05-1.53).

With the increased complexity of breast cancer care delivery, there is concern that advances in clinical outcomes may be blunted by the challenges in delivering timely care, particularly when interdisciplinary coordination is required. Over the past decade, delays in breast cancer treatment have increased nationally, ${ }^{19-21}$ with results from a multiinstitutional cohort study demonstrating an increase in median time from diagnosis to chemotherapy initiation from 10.8 in 2003 to 13.3 weeks in 2009. ${ }^{21}$ Multiple factors, including age, disease-specific characteristics, type of surgical procedure, and additional testing have been found to influence this trend. ${ }^{21-27}$ In an era of increasing use of Oncotype DX to assist with chemotherapy treatment decisions, an aging population with comorbidities needing to be addressed, and the more prevalent use of MIR, it is not surprising that wait times to adjuvant therapy continue to increase.

Although our data suggest that DF/BWCC performs reasonably well, with approximately three-quarters of patients initiating therapy within 6 weeks of surgery, an analysis of patients with a higher risk of recurrence demonstrated that $21.4 \%$ of patients $(15 / 70)$ with stage III tumors, $20.2 \%$ of patients (21/104) with TNBC, and $29.5 \%$ of patients (46/156) with HER2positive breast cancer initiated chemotherapy more than 6 weeks from LDS. These findings highlight opportunities for improvement.

Notably, we identified LDS as a significant factor associated with UCD, with patients undergoing MIR being more likely to experience delay, a finding that remained significant in patients with stage II-III disease. These results may be attributed to longer recovery time for MIR, including time for wound healing and drain removal, and the increased risk of postoperative complications. In prior work, we found 
Losk et al

\begin{tabular}{|c|c|c|c|}
\hline \multirow[b]{2}{*}{ Characteristics } & $\begin{array}{l}\text { All Patients } \\
(\mathrm{N}=523)\end{array}$ & $\begin{array}{c}29-41 \text { Days } \\
(n=223)\end{array}$ & $\begin{array}{c}\geq 42 \text { Days } \\
(n=141)\end{array}$ \\
\hline & n (\%) & $\begin{array}{c}\text { OR } \\
(95 \% \mathrm{Cl})\end{array}$ & $\begin{array}{c}\text { OR } \\
(95 \% \text { Cl) }\end{array}$ \\
\hline \multicolumn{4}{|l|}{ Age, y } \\
\hline$<40$ & $68(13.0 \%)$ & $0.58(0.29-1.15)$ & $0.64(0.28-1.46)$ \\
\hline $40-49$ & $165(31.5 \%)$ & 1.00 & 1.00 \\
\hline 50-59 & $150(28.7 \%)$ & $0.91(0.53-1.56)$ & $1.68(0.89-3.20)$ \\
\hline $60-69$ & $113(21.6 \%)$ & $1.83(0.94-3.58)$ & $2.92(1.34-6.36)$ \\
\hline$\geq 70$ & $27(5.2 \%)$ & $0.94(0.29-3.08)$ & $2.96(0.86-10.21)$ \\
\hline \multicolumn{4}{|l|}{ Race } \\
\hline White & $424(81.1 \%)$ & 1.00 & 1.00 \\
\hline Nonwhite & $79(15.1 \%)$ & $0.63(0.34-1.15)$ & $1.05(0.55-2.04)$ \\
\hline Missing & $20(3.8 \%)$ & $0.39(0.12-1.27)$ & $0.78(0.23-2.62)$ \\
\hline \multicolumn{4}{|l|}{ Primary insurance } \\
\hline Private & $419(80.1 \%)$ & 1.00 & 1.00 \\
\hline Public & $104(19.9 \%)$ & $1.05(0.55-2.03)$ & $1.61(0.79-3.29)$ \\
\hline \multicolumn{4}{|l|}{ Year of surgery } \\
\hline 2011 & $111(21.2 \%)$ & 1.00 & 1.00 \\
\hline 2012 & $207(39.6 \%)$ & $0.75(0.31-1.35)$ & $0.60(0.30-1.18)$ \\
\hline 2013 & $205(39.2 \%)$ & $0.74(0.41-1.33)$ & $0.78(0.40-1.52)$ \\
\hline \multicolumn{4}{|l|}{ Stage } \\
\hline 1 & $215(41.1 \%)$ & 1.00 & 1.00 \\
\hline II & $238(45.5 \%)$ & $1.14(0.71-1.84)$ & $1.13(0.65-1.96)$ \\
\hline III & $70(13.4 \%)$ & $0.93(0.46-1.89)$ & $0.44(0.19-1.03)$ \\
\hline \multicolumn{4}{|l|}{ Grade } \\
\hline 1 & $19(3.6 \%)$ & 1.00 & 1.00 \\
\hline 2 & $181(34.6 \%)$ & $1.51(0.45-5.04)$ & $1.09(0.29-4.07)$ \\
\hline 3 & $323(61.8 \%)$ & $1.21(0.37-4.03)$ & $0.89(0.24-3.31)$ \\
\hline \multicolumn{4}{|l|}{ Tumor subtype } \\
\hline HR+/HER2- & $263(50.3 \%)$ & $1.74(0.94-3.21)$ & $2.71(1.28-5.73)$ \\
\hline HER2+ & $156(29.8 \%)$ & $1.05(0.57-1.92)$ & $1.78(0.86-3.68)$ \\
\hline HR-/HER2- & $104(19.9 \%)$ & 1.00 & 1.00 \\
\hline \multicolumn{4}{|l|}{ Type of surgery } \\
\hline Lumpectomy & $265(50.7 \%)$ & 1.00 & 1.00 \\
\hline Mastectomy & $89(17.0 \%)$ & $1.16(0.62-2.18)$ & $3.32(1.68-6.56)$ \\
\hline $\begin{array}{l}\text { Mastectomy with } \\
\text { reconstruction }\end{array}$ & $169(32.3 \%)$ & $1.99(1.17-3.36)$ & $5.09(2.77-9.34)$ \\
\hline \multicolumn{4}{|l|}{ Pathology sign-out } \\
\hline Within 10 days & 366 (70.0\%) & 1.00 & 1.00 \\
\hline$>10$ days & $157(30.0 \%)$ & $1.38(0.85-2.26)$ & $2.04(1.19-3.50)$ \\
\hline
\end{tabular}

Reference group defined as "chemotherapy start within 28 days after last definitive surgery."

Abbreviations: HR, hormone receptor; OR, odds ratio.

that these patients also frequently experienced delays from initial consultation to breast surgery due to complexities in decision-making regarding type of reconstruction and challenges in operating room scheduling. ${ }^{14}$ Our data raise questions about how to balance long-term quality-of-life and cosmetic outcomes with the potential impact of delays in chemotherapy on clinical outcomes. Even if surgical scheduling could be optimally streamlined, the complexity and potential delays of patient decision-making (in

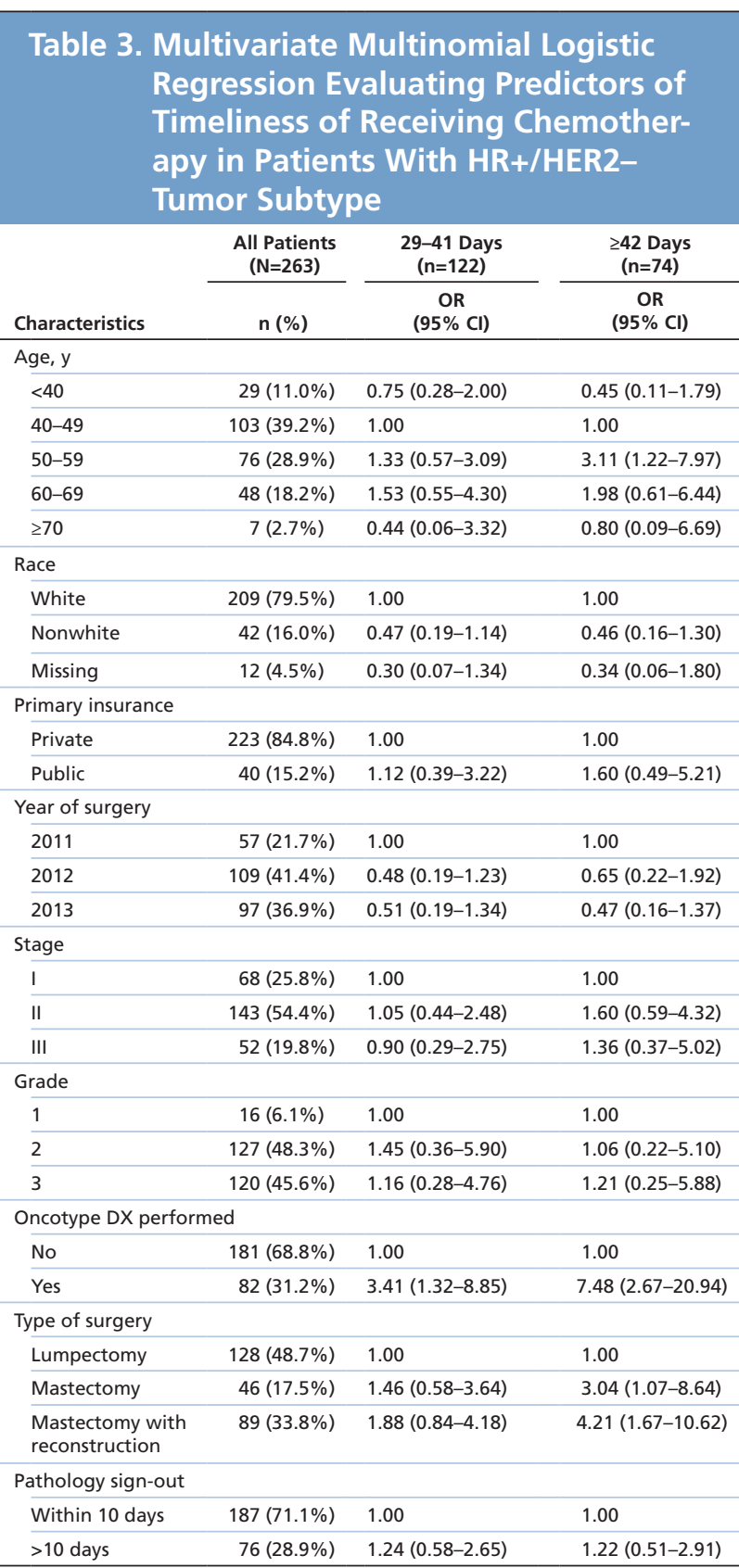

Reference group defined as "chemotherapy start within 28 days after LDS."

Abbreviations: HR, hormone receptor; OR, odds ratio.

terms of the surgical procedure), as well as wound healing requirements, will continue to make timely initiation of chemotherapy a challenge in this setting. This raises the question of whether preoperative chemotherapy should be more frequently considered in patients for whom chemotherapy is clearly indicated based on preoperative clinical staging and who desire to undergo MIR. 
Delays to Chemotherapy in Breast Cancer

\begin{tabular}{|c|c|c|c|}
\hline \multirow[b]{2}{*}{ Characteristics } & $\begin{array}{l}\text { All Patients } \\
(\mathrm{N}=308)\end{array}$ & $\begin{array}{c}29-41 \text { Days } \\
(n=135)\end{array}$ & $\begin{array}{c}\geq 42 \text { Days } \\
(\mathrm{n}=84)\end{array}$ \\
\hline & n (\%) & $\begin{array}{c}\text { OR } \\
(95 \% \mathrm{Cl})\end{array}$ & $\begin{array}{c}\text { OR } \\
(95 \% \mathrm{Cl})\end{array}$ \\
\hline \multicolumn{4}{|l|}{ Age, y } \\
\hline$<40$ & $41(13.3 \%)$ & $0.62(0.26-1.49)$ & $0.58(0.20-1.64)$ \\
\hline $40-49$ & $98(31.8 \%)$ & 1.00 & 1.00 \\
\hline $50-59$ & $89(28.9 \%)$ & $0.75(0.36-1.53)$ & $1.57(0.70-3.49)$ \\
\hline 60-69 & $62(20.1 \%)$ & $1.44(0.59-3.50)$ & $1.66(0.60-4.59)$ \\
\hline$\geq 70$ & $18(5.9 \%)$ & $1.55(0.37-6.55)$ & $2.15(0.45-10.37)$ \\
\hline \multicolumn{4}{|l|}{ Race } \\
\hline White & $243(78.9 \%)$ & 1.00 & 1.00 \\
\hline Nonwhite & $52(16.9 \%)$ & $0.69(0.32-1.48)$ & $1.11(0.49-2.50)$ \\
\hline Missing & $13(4.2 \%)$ & $0.39(0.10-1.53)$ & $0.50(0.11-2.33)$ \\
\hline \multicolumn{4}{|l|}{ Primary insurance } \\
\hline Private & $245(79.6 \%)$ & 1.00 & 1.00 \\
\hline Public & $63(20.4 \%)$ & $1.01(0.43-2.34)$ & $1.50(0.60-3.74)$ \\
\hline \multicolumn{4}{|l|}{ Year of surgery } \\
\hline 2011 & $58(18.8 \%)$ & 1.00 & 1.00 \\
\hline 2012 & $126(40.9 \%)$ & $0.52(0.23-1.16)$ & $0.49(0.20-1.23)$ \\
\hline 2013 & $124(40.3 \%)$ & $0.70(0.31-1.61)$ & $0.83(0.33-2.06)$ \\
\hline \multicolumn{4}{|l|}{ Grade } \\
\hline 1 & $14(4.5 \%)$ & 1.00 & 1.00 \\
\hline 2 & $124(40.3 \%)$ & $1.58(0.40-6.23)$ & $1.04(0.23-4.84)$ \\
\hline 3 & $170(55.2 \%)$ & $0.91(0.24-3.53)$ & $0.84(0.19-3.79)$ \\
\hline \multicolumn{4}{|l|}{ Type of surgery } \\
\hline Lumpectomy & $143(46.4 \%)$ & 1.00 & 1.00 \\
\hline Mastectomy & $65(21.1 \%)$ & $0.83(0.40-1.74)$ & $2.01(0.90-4.48)$ \\
\hline $\begin{array}{l}\text { Mastectomy with } \\
\text { reconstruction }\end{array}$ & $100(32.5 \%)$ & $1.53(0.77-3.04)$ & $3.87(1.76-8.49)$ \\
\hline \multicolumn{4}{|l|}{ Pathology sign-out } \\
\hline Within 10 days & $225(73.1 \%)$ & 1.00 & 1.00 \\
\hline$>10$ days & $83(26.9 \%)$ & $0.90(0.47-1.70)$ & $1.16(0.58-2.33)$ \\
\hline
\end{tabular}

Reference group defined as "chemotherapy start within 28 days after LDS."

Abbreviation: OR, odds ratio.

We also found that patients with HR-positive/ HER2-negative disease were more likely to experience delays in chemotherapy initiation compared with those with TNBC. It is possible, given the more biologically aggressive nature of TNBC, that physicians attempt to expedite processes for these patients to initiate treatment more quickly. Another explanation is that the decision to recommend chemotherapy is more straightforward with TNBC, than with HR-positive/HER2-negative tumors. In support of this hypothesis, Oncotype DX testing was ordered in $31.2 \%$ of patients with HR-positive/HER2-negative tumors. In our subsequent analyses, we found that Oncotype DX testing increased the likelihood of
UCD by more than 7 -fold. Vandergrift et $\mathrm{al}^{21}$ report similar findings; in their study, Oncotype DX testing was associated with a 2.2-week increase in time to chemotherapy initiation $(P<.001)$.

The only sociodemographic characteristic found to be associated with UCD was older age, with patients between 60 and 69 years of age being more likely to experience delays. This finding could be due to comorbidities among older patients, a factor not captured in our analyses, or due to different consideration of older patients, weighing the risks and benefits of adjuvant chemotherapy. It is also possible that providers are more apt to push for rapid treatment in younger patients; however, we were unable to assess this based on our analyses.

Our overall goal in conducting these analyses was to identify specific barriers to timely care in order to optimally focus our interventional efforts. Based on this analysis, we have initiated several efforts, including improving pathology turnaround time, expediting the communication of pathology results to providers, reducing the time from surgery to postoperative medical oncology appointment, and developing criteria for reflex ordering of Oncotype DX testing. We plan to examine the impact of these interventions on the timing of chemotherapy initiation in future studies.

Our study has several limitations. First, the relative importance of the barriers we identified may not be generalizable to other settings, particularly non-comprehensive cancer centers. Our surgeons and medical oncologists practice within the same center, and use shared electronic medical record and communication systems. The challenges of care coordination may be even greater in settings where surgery and medical oncology do not share this close relationship. Second, although we conducted chart review audits to elucidate additional factors associated with increased wait times, in half of those cases we were unable to identify a specific cause for the delayed care. Our data did not capture comorbidities of the patient cohort, which may play an important role in chemotherapy timing. Lastly, we were unable to analyze factors specifically associated with delays in TNBC and HER2-positive disease, subtypes for which the impact of delays may be greatest. Future research is warranted to uncover additional factors associated with delays, particularly in specific disease subtypes. Despite these limitations, our analysis has 
Losk et al

identified several areas of opportunity that are currently being addressed.

\section{Conclusions}

With the increase in wait times for breast cancer treatment, cancer centers should engage in efforts to measure and improve the timeliness of care, with a particular focus on modifiable factors within provider and institutional control.

\section{References}

1. Allemani C, Weir HK, Carreira $H$, et al. Global surveillance of cancer survival 1995-2009: analysis of individual data for 25,676,887 patients from 279 population-based registries in 67 countries (CONCORD-2). Lancet 2015;385:977-1010.

2. Berry DA, Cronin KA, Plevritis SK, et al. Effect of screening and adjuvant therapy on mortality from breast cancer. N Engl J Med 2005;353:17841792 .

3. DeSantis CE, Bray F, Ferlay J, et al. International variation in female breast cancer incidence and mortality rates. Cancer Epidemiol Biomarkers Prev 2015;24:1495-1506.

4. Saadatmand $S$, Bretveld R, Siesling $S$, et al. Influence of tumour stage at breast cancer detection on survival in modern times: population based study in 173,797 patients. BMJ 2015;351:h4901.

5. Berry DA, Cirrincione C, Henderson IC, et al. Estrogen-receptor status and outcomes of modern chemotherapy for patients with node-positive breast cancer. JAMA 2006;295:1658-1667.

6. Early Breast Cancer Trialists' Collaborative Group (EBCTCG). Effects of chemotherapy and hormonal therapy for early breast cancer on recurrence and 15-year survival: an overview of the randomised trials. Lancet 2005;365:1687-1717.

7. Chavez-MacGregor M, Clarke CA, Lichtensztajn DY, et al. Delayed initiation of adjuvant chemotherapy among patients with breast cancer. JAMA Oncol 2016;2:322-329.

8. Colleoni M, Gelber RD. Time to initiation of adjuvant chemotherapy for early breast cancer and outcome: the earlier, the better? J Clin Oncol 2014;32:717-719.

9. Farolfi A, Scarpi E, Rocca A, et al. Time to initiation of adjuvant chemotherapy in patients with rapidly proliferating early breast cancer. Eur J Cancer 2015;51:1874-1881.

10. Gagliato Dde M, Gonzalez-Angulo AM, Lei X, et al. Clinical impact of delaying initiation of adjuvant chemotherapy in patients with breast cancer. J Clin Oncol 2014;32:735-744.
11. Lohrisch C, Paltiel C, Gelmon K, et al. Impact on survival of time from definitive surgery to initiation of adjuvant chemotherapy for early-stage breast cancer. J Clin Oncol 2006;24:4888-4894.

12. Trufelli DC, de Matos LL, Santi PX, et al. Adjuvant treatment delay in breast cancer patients. Rev Assoc Med Bras 2015;61:411-416.

13. Yu KD, Huang $S$, Zhang JX, et al. Association between delayed initiation of adjuvant CMF or anthracycline-based chemotherapy and survival in breast cancer: a systematic review and meta-analysis. BMC Cancer 2013;13:240.

14. Golshan M, Losk K, Kadish S, et al. Understanding process-of-care delays in surgical treatment of breast cancer at a comprehensive cancer center. Breast Cancer Res Treat 2014;148:125-133.

15. Senkus E, Kyriakides S, Penault-Llorca F, et al. Primary breast cancer: ESMO Clinical Practice Guidelines for diagnosis, treatment and followup. Ann Oncol 2013;24(Suppl 6):vi7-23.

16. Desch CE, McNiff KK, Schneider EC, et al. American Society of Clinical Oncology/National Comprehensive Cancer Network quality measures. J Clin Oncol 2008;26:3631-3637.

17. Cold S, During M, Ewertz M, et al. Does timing of adjuvant chemotherapy influence the prognosis after early breast cancer? Results of the Danish Breast Cancer Cooperative Group (DBCG). Br J Cancer 2005;93:627-632.

18. Shannon C, Ashley S, Smith IE. Does timing of adjuvant chemotherapy for early breast cancer influence survival? J Clin Oncol 2003;21:3792-3797.

19. Bleicher RJ, Ruth $K$, Sigurdson ER, et al. Time to surgery and breast cancer survival in the United States. JAMA Oncol 2016;2:330-339.

20. Liederbach E, Sisco M, Wang C, et al. Wait times for breast surgical operations, 2003-2011: a report from the National Cancer Data Base. Ann Surg Oncol 2015;22:899-907.

21. Vandergrift JL, Niland JC, Theriault RL, et al. Time to adjuvant chemotherapy for breast cancer in National Comprehensive Cancer Network institutions. J Natl Cancer Inst 2013;105:104-112.

22. Alderman AK, Collins ED, Schott A, et al. The impact of breast reconstruction on the delivery of chemotherapy. Cancer 2010;116:17911800.

23. Barry PN, Riley EC, Pan J, et al. Delay of adjuvant chemotherapy after elective mastectomy and immediate reconstruction in breast-conservation candidates: a matched-pair analysis. Am J Clin Oncol 2014;37:575-579.

24. Fedewa SA, Ward EM, Stewart AK, et al. Delays in adjuvant chemotherapy treatment among patients with breast cancer are more likely in African American and Hispanic populations: a national cohort study 2004-2006. J Clin Oncol 2010;28:4135-4141.

25. Freedman RA, He Y, Winer EP, et al. Racial/Ethnic differences in receipt of timely adjuvant therapy for older women with breast cancer: are delays influenced by the hospitals where patients obtain surgical care? Health Serv Res 2013;48:1669-1683.

26. Lee J, Lee SK, Kim S, et al. Does immediate breast reconstruction after mastectomy affect the initiation of adjuvant chemotherapy? J Breast Cancer 2011;14:322-327.

27. Sharpe SM, Liederbach E, Czechura T, et al. Impact of bilateral versus unilateral mastectomy on short term outcomes and adjuvant therapy, 2003-2010: a report from the National Cancer Data Base. Ann Surg Oncol 2014;21:2920-2927. 